\title{
Mobile Task Management for Medical Ward Rounds - The MEDo Approach
}

\author{
Rüdiger Pryss, David Langer, Manfred Reichert and Alena Hallerbach \\ Institute of Databases and Information Systems, Ulm University, Germany \\ \{ruediger.pryss, manfred.reichert\}@uni-ulm.de, \\ mail@davidlanger.de, alena.h@llerba.ch
}

\begin{abstract}
In hospitals, ward rounds are crucial for decision-making in the context of patient treatment processes. In the course of a ward round, new tasks are defined and allocated to physicians and nurses. In clinical practice, however, these tasks are not systematically managed. During ward rounds, they are jotted down using pen and paper, and their later processing is prone to errors. Furthermore, medical staff must keep track of the processing status of its tasks (e.g., medical orders). To relieve staff members from such a manual task management, the MEDo approach supports ward rounds by transforming the pen and paper worksheet to a mobile user interface on a tablet integrating process support, mobile task management, and access to the electronic patient record. Interviews we conducted have confirmed that medical staff craves for mobile task and process support on wards. Furthermore, in several user experiments, we have proven that MEDo puts task acquisition on a level comparable to that of pen and paper. Overall, with MEDo, physicians can create, monitor and share tasks using a mobile and user-friendly platform.
\end{abstract}

Key words: Mobility, Healthcare, Mobile Task Management

\section{Introduction}

In the context of clincial ward rounds, there is a high demand for improving interactions and communication among healthcare professionals. Problems of ward inpatients become more and more complex and managing patient data directly at the bedside is a must. Although existing technology tailored to clinical demands has reached a mature level, still there is a lack of digital task support during ward rounds. To better understand how such a support can be smoothly provided, we attended numerous ward rounds, interviewed medical staff, and considered existing solutions established in clinical practice. Our findings have raised two major issues clinicians sorely need. First of all, the paper-based task worksheet shall be transferred to a mobile and digital variant. Clinicians use such worksheets as their personal information system to organize their tasks. In turn, data gathered with this sheet is not related to the one of the hospital information system. For example, for adding a new task to his sheet during a ward round, a physician will make a note like "Mrs.Richards: X-ray request arranged, monitor 
status and check images today". In the pen and paper version, basically, each task description solely consists of the patient's name and free text. Therefore, another demand posed by clinicians is to enhance task management with both workflow support (e.g., to keep track of medical orders) and integrated access to patient data. With MEDo ${ }^{1}$ (MedicalDo), we target at a process-aware, mobile task support of the medical staff during ward rounds. This paper reports on the experiences we gathered with MEDo during a number of clinical ward rounds. The remainder of this paper is organized as follows: Section 2 describes the method we applied to understand the requirements of medical ward rounds. In Section 3, we share the experiences we gathered with MEDo and discuss our lessons learned. Section 4 discusses related work and Section 5 concludes with an outlook.

\section{MEDo Pre-Phase: Ward Round Investigation}

We started our clinical investigations with a survey. In particular, we evaluated how physicians perceive the current management of ward rounds when using pen and paper. First, we revealed how physicians perceive task acquisition. Second, we asked them how they currently manage ward rounds in general, i.e., the communication with other healthcare professionals or the access to patient information provided by any hospital information system. Fig. 1 depicts the results of this survey. Later, we will compare the use of pen and paper in the context of a ward round with the one of MEDo.

\begin{tabular}{lcc}
\hline Question & Mean Value & Standard Deviation \\
\hline Task Definition & 2.22 & 1.30 \\
Ward Round Management & 2.50 & 1.12 \\
\hline \multicolumn{2}{c}{ | Scale from 1 to 6 | 1: Best Value | 6: Worst Value | } \\
\hline
\end{tabular}

Fig. 1. MEDo Pre-Phase Evaluation

To identify and capture relevant requirements issues and to understand how they are currently addressed, we analyzed several ward rounds. More precisely, we participated in four wards rounds at different clinical departments. The basic facts related to these ward rounds are summarized in Fig. 2. Interestingly, only one clinical department already provides IT support for accessing patient information during ward rounds; i.e., imaging data and laboratory results can be accessed during the ward round using a tablet PC. As can be further seen, the ward rounds we analyzed vary significantly in respect to their characteristics (cf. Fig. 2).

Based on these insights, we extracted procedures performed or triggered in the context of a ward round. In particular, we were interested in how they can

\footnotetext{
${ }^{1}$ MEDo video under http://apps.dbis.info/medo/medo.mov
} 


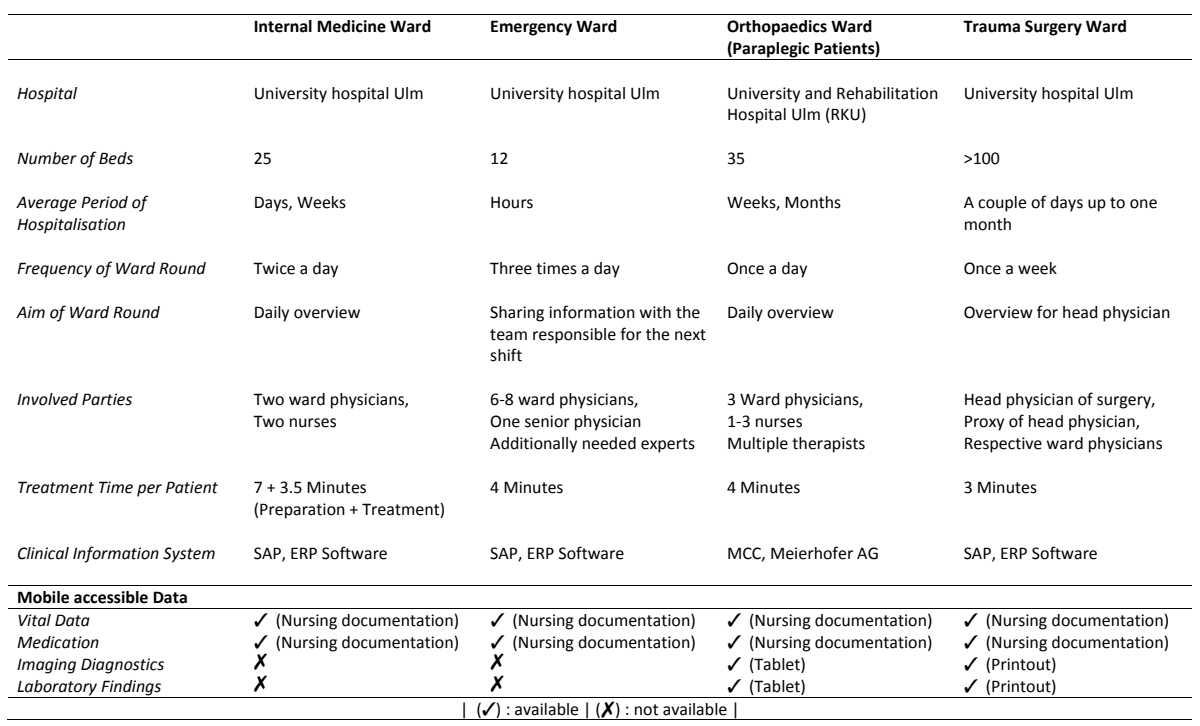

Fig. 2. Basic Facts Characterizing the Investigated Ward Rounds

be smoothly integrated with mobile task assistance. Additionally, we identified the patient data physicians want to access in the context of their task lists. In order to identify required procedures and needed patient information, we attended the four different ward rounds several times and then transfered the insights we gained to a more formal IT representation. In particular, explicitly modeling the identified procedures in terms of BPMN has proven to be useful; i.e., BPMN models provided a good basis for discussing the procedures in the context of a ward round with physicians. Relevant patient information, medical staff quickly wants to access is depicted in Fig. 3a. Additionally, Fig. 3b shows the data privileges required by physicians and nurses in this context. It is noteworthy that physicians want to share their task sheets with nurses and colleagues in order to improve communication.

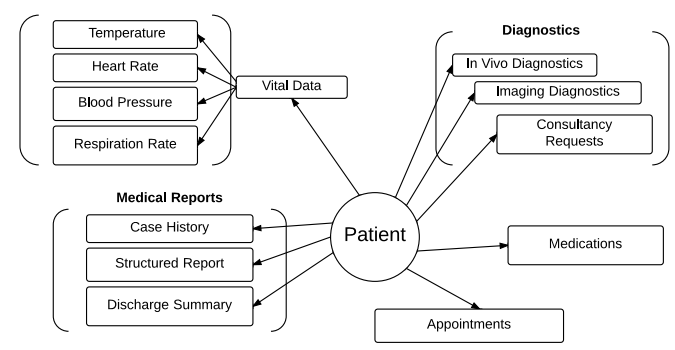

(a) Patient Information Data

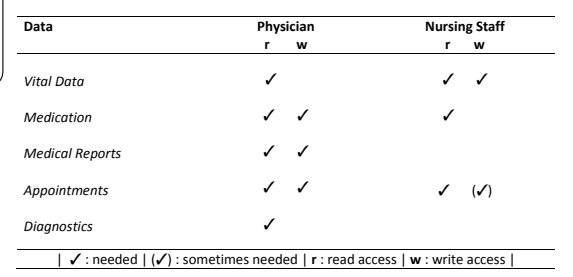

(b) Access Privileges

Fig. 3. Patient Information and Access Privileges 
We present two wards rounds in detail.

\subsection{Ward Rounds in Internal Medicine}

Fig. 4 shows the process for coordinating the steps of a ward round in a department of internal medicine. This type of ward round is common to many hospitals and encompasses two stages. In the first stage, the physician analyzes the cases of all inpatients without seeing the patients. For this purpose, he accesses the hospital information system to retrieve information about the inpatients. Further, he is assisted by a nurse and receives information from her. Finally, relevant issues and alternatives regarding patient treatment are discussed in this first phase. In the second phase, the physician visits the inpatients, makes notes using pen and paper, and verifies his decisions. If new tasks (e.g., medical orders) emerge, he makes a note on his worksheet and adopts this change in the hospital information systems afterwards. Examples of tasks and workflows, respectively, emerging in the context of the second phase include requests for X-ray examinations, laboratory tests, consultancy requests, and changes in patient medication. Making appointments with external hospital departments constitutes another kind of task emerging in the context of a ward round. In summary, task worksheets based on pen and paper are crucial for ward rounds in internal medicine.

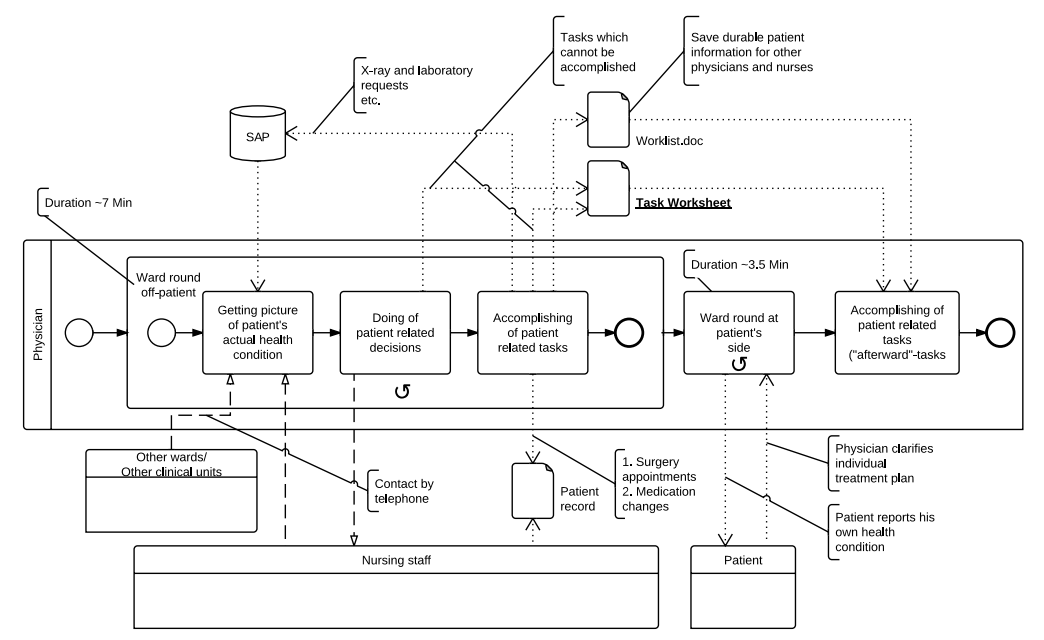

Fig. 4. Two-phased Process Related to Ward Rounds in Internal Medicine

\subsection{Ward Rounds in Orthopaedic Medicine}

Fig. 5 shows the basic procedure of the ward round in orthopaedic medicine we accompanied. In particular, no mobile access to the complete electronic patient 
record is provided. However, the preparation of certain patient-related tasks can be accomplished by using a tablet PC, which provides mobile access to selected parts of the hospital information system (HIS). Although, the functions provided by this HIS are not adequately mapped to the tablet, physicians may order certain examinations based on this mobile user interface. Still, most orders are processed in a paper-based fashion afterwards. Interestingly, physicians considered this mobile way of acquiring tasks and making medical orders as useful, and emphasized that it contributes to reduce error rates (e.g., ommissions). Again, the paper-based task worksheet constitutes the most prevalent instrument for memorizing and communicating upcoming tasks. During a ward round, physicians make notes about upcoming tasks and after the ward round they start processing them. For this scenario, we identified more or less the same workflows as for the other two ward rounds depicted in Fig. 2. However, requests for external appointments are not required due to the crucial health status of the patients.

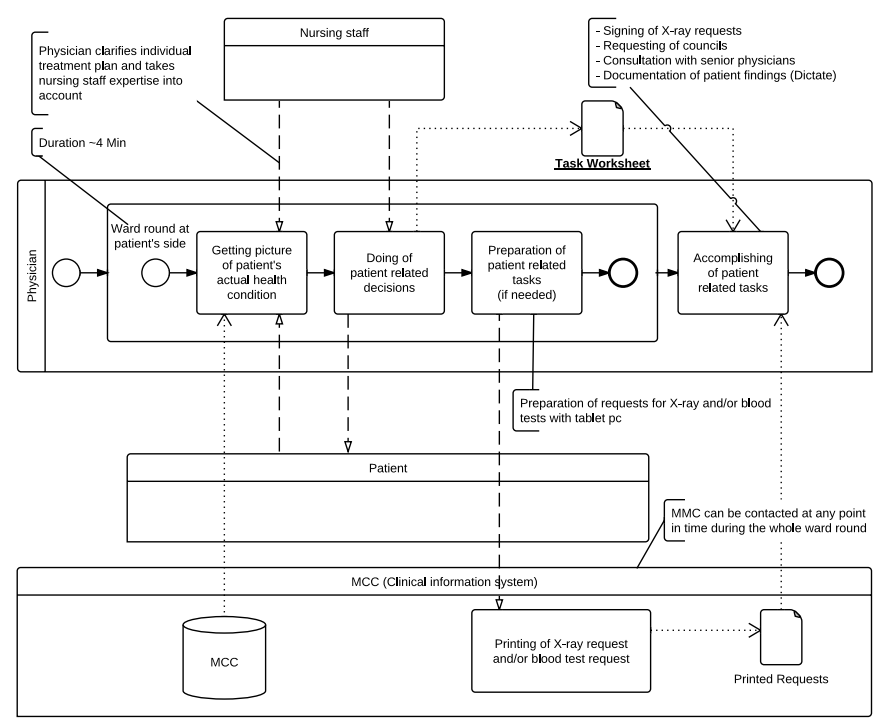

Fig. 5. Process for Ward Rounds in Orthopaedic Medicine

When analyzing the other two ward round types depicted in Fig. 2, basically, we identified the same or similar workflows for implementing core tasks. Based on the overall experiences gathered during the analysis of the different ward round scenarios, we are able to define fundamental requirements to be met by any mobile task support during a ward round. We considered these requirements when designing the MEDo prototype. 


\section{MEDo Findings}

Before designing and implementing MEDo, we evaluated existing mobile tablet frameworks to select the one meeting our requirements best. In this context, aspects like size, weight, display resolution, viewing angle dependency, and input sensibility were considered. Based on these properties, we deciced to develop the MEDo proof-of-concept for mobile task management on the iPad. Our findings with MEDo are presented along three dimensions. First, we discuss findings related to digital task creation and management. Second, we give insights related to the workflows supporting respective tasks. Third, we discusss findings related to the information architecture (i.e., the user interface and control flow structure) we realized in MEDo.

\subsection{Task Creation and Management}

We first summarize the requirements raised by physicians with respect to worksheets enabling mobile task management. We derived these requirements by attending ward rounds and interviewing medical staff. Usually, physicians and nurses organize their pending and daily tasks based on paper worksheets. Thereby, task definition is always accomplished the same way. For example, physicians make handwritten notes on their worksheet, whereas each note consists of a patient name and descriptive free text. Creating and managing tasks this way means being quick and flexible. Thus, tasks can be acquired in different context and task acquisition can be interrupted at any time. Exactly these two aspects have been mentioned by physicians as major reasons for still using pen and paper. Hence, when transferring task management to a mobile IT application, one must ensure that its use is time-efficient and intuitive, offering the same flexibility as pen and paper. In particular, usability can be improved using results from cognitive science and design techniques from usability engineering, like choosing the right colours or realizing a comprehensible and useful segmentation of the application screen. Interviews and usability tests with medical staff helped us to figure out what elements are intuitive for them. Fig. 6 summarizes major requirements for mobile task creation and management.

\begin{tabular}{|c|c|c|}
\hline & Task Requirements & Description \\
\hline $\mathbf{R}_{1}$ & Manage the task digitally with mobile assistance & $\begin{array}{l}\text { Provide patient's name and free text to physicians in order to manage their digital task } \\
\text { entries (todo-items) comparable to that of pen and paper. }\end{array}$ \\
\hline $\mathbf{R}_{\mathbf{2}}$ & Access patient information contextually linked to tasks & $\begin{array}{l}\text { Provide quick access to patient information, e.g., vital data, medical reports, external } \\
\text { appointments, medications, and diagnostics. }\end{array}$ \\
\hline \multirow[t]{4}{*}{$\mathbf{R}_{3}$} & Ensure a high input speed for task acquisition & Provide high input speed for the task definition. \\
\hline & $\mathbf{R}_{31}$ Provide text templates for the task creation & Provide pre-defined text templates to physicians for creating tasks quickly. \\
\hline & $\mathbf{R}_{32}$ Enable voice recording for the task creation & Provide voice recording feature to physicians for executing tasks comfortably. \\
\hline & $\mathbf{R}_{33}$ Enable the creation of new text templates & Enable physicians to create new or personally tailored text templates. \\
\hline $\mathbf{R}_{4}$ & Enable barcode scanning for medications, plasters, bandages, etc. & $\begin{array}{l}\text { Provide nurses with a barcode feature to scan medications, plasters, bandages, or to } \\
\text { dress material used for patients. }\end{array}$ \\
\hline $\mathbf{R}_{\mathbf{5}}$ & & Provide a filter function to physicians to save time while managing task entries. \\
\hline & Organize tasks according to their importance & Provide physicians with a feature to organize tasks according to their importance. \\
\hline
\end{tabular}

Fig. 6. Requirements for Mobile Task Creation and Management

Overall, when meeting these requirements, tasks can be created quickly and smoothly integrated with patient information. However, regarding our first pro- 
totype it has turned out that task acquisition was perceived as not being fast enough. To reach a level of usability comparable to that of pen and paper, therefore, further improvements became necessary (cf. $R_{31}-R_{33}$ in Fig. 6). Among others, we realized a collection of text templates that may be used when creating tasks. Our user tests have shown that these text templates have increased task acquisition speed significantly. Furthermore, tasks may be acquired and created using the voice recording feature we integrated with MEDo.

\subsection{Workflow Management}

Flexible workflow support [1] is another key requirement raised by physicians. By integrating mobile task assistance with workflows, it shall become possible for physicians to easily keep track of their tasks (e.g., medical orders), get aggregated overview lists, or be immediatly notified whenever any problems occurs. For example, the state of an X-ray examination, requested during a ward round, can be easily monitored based on the corresponding workflow. Fig. 7 depicts an example of such a workflow.

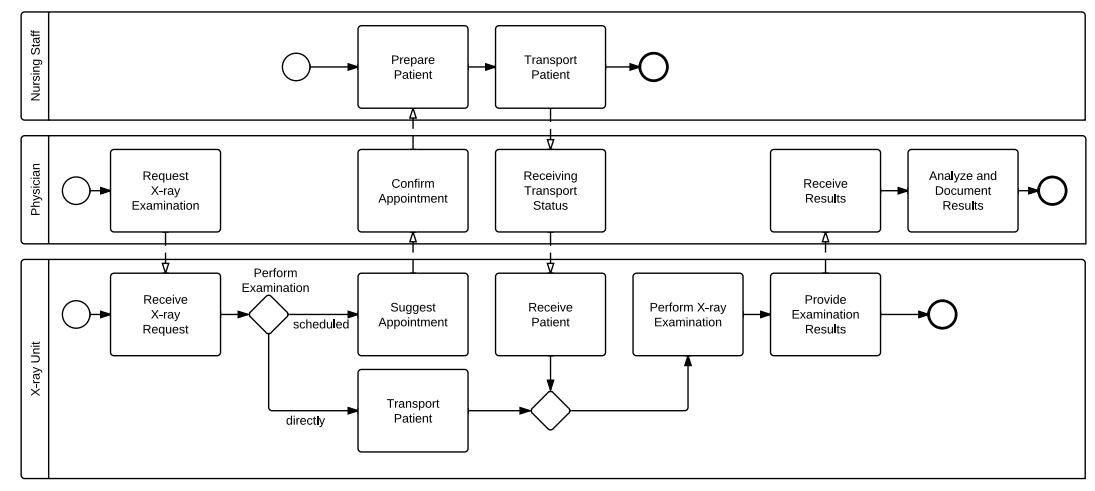

Fig. 7. Process for Handling X-ray Examinations

Concerning workflow-based task support, we gathered three major requirements from physicians. First, they asked for a quick overview of tasks and related workflows (cf. $\mathrm{R}_{9}$ ). Second, these workflows shall be automatically triggered and coordinated by a process-aware HIS when defining tasks ( $\mathrm{cf} . \mathrm{R}_{7}$ ). Third, the current state of a task and its underlying workflow, respectively, shall be accessible and changeable based on different user views $\left(c f . R_{8}, R_{9}\right)$. For example, if the results of a laboratory order arrive, the physician will get immediate access to them. Afterwards, he may change the state of this task and its related workflow to "seen". In particular, this user interaction is possible from different views in MEDo. For example, the physician may change the status of her task by using the task overview or laboratory view. Fig. 8 summarizes the requirements related to workflow-based task support in MEDo. 


\begin{tabular}{|c|c|c|}
\hline & Workflow Requirements & Description \\
\hline $\mathbf{R}_{7}$ & Use workflows to keep track of the status of tasks & $\begin{array}{l}\text { Support workflows for handling and processing tasks and integrate these workflows } \\
\text { smoothly with the hospital information system. }\end{array}$ \\
\hline $\mathbf{R}_{8}$ & Interrupt tasks and continue them later & $\begin{array}{l}\text { Workflows as well as user interfaces must enable healthcare professionals to interrupt } \\
\text { their current work at any point in time and to continue it later. }\end{array}$ \\
\hline$R_{9}$ & Provide personalized views on workflows to users & The workflows must be intuitively presented to healthcare professionals. \\
\hline
\end{tabular}

Fig. 8. Workflow Requirements

\subsection{Information Architecture}

Another fundamental aspect of any digital task worksheet is to identify how the information architecture (i.e., the fundamental control flow structure of the user interface) shall look like. For example, it is compelling to allow users to interrupt a task at any point in time and to continue it later. In practice, this means that users must be able to switch between different views within the mobile application. To identify which views and context switches are actually needed, we identified scenarios covering task acquisition and execution, characteristic types of tasks, and situations requiring context switches. Fig. 9 exemplarily depicts three task creating scenarios.

\begin{tabular}{cl}
\hline Scenario & Description \\
\hline Scenario I & $\begin{array}{l}\text { After a ward round, the physician wants to see all upcoming tasks of the day at a glance. He then uses this overview for } \\
\text { planning his work day (cf. Fig. 11a). }\end{array}$ \\
Scenario II & $\begin{array}{l}\text { During a ward round, the physician wants to know whether an X-ray examination has already been requested and } \\
\text { what status the examination currently has. Fig. 11b shows that the X-ray has arrived and the physicians can finish the } \\
\text { workflow by setting the state to Mark as seen. }\end{array}$ \\
$\begin{array}{l}\text { During a ward round, a set of tasks shall be created. Often, a particular physician makes orders, while another one is } \\
\text { Scenario III } \\
\text { collecting them. Fig. 12a shows the creation of a task using text templates, whereas Fig. 12b shows the creation of a } \\
\text { laboratory request using a specialized laboratory creation view. }\end{array}$ \\
\hline
\end{tabular}

Fig. 9. Example Scenarios

The information architecture realized in MEDo is depicted in Fig. 10. Most important, tasks constitute the predominant paradigm for user interaction. In particular, the physician does not need to switch her current view if she wants to create a new task. For example, a request for an X-ray examination may be entered using the task view or alternatively within every other view (e.g., the laboratory view). Due to lack of space, we only present selected features of the MEDo information architecture. In particular, we want to give some impressions related to the scenarios described in Fig. 9 and discuss how they are realized in MEDo. Fig. 11a shows the MEDo entry view. Marking 1 refers to the list of all patients. In MEDo, patients may either be listed alphabetically or according to the rooms they are assigned to.

Marking 2 refers to both upcoming and recently completed tasks. In turn, Marking 3 shows the processing state of a selected task (X-ray request). For example, when the $\mathrm{X}$-ray images arrive, a symbol change notifies the physician about this status change. To study the results obtained, in turn, she may switch to the imaging view depicted in Fig. 11b. Marking \& refers to an element allowing the physician to change the state of the X-ray request to "finished". Finally, the corresponding workflow is completed. Marking 5 in Fig. 12a shows the predefined text templates the physician can use when defining a task. This feature 


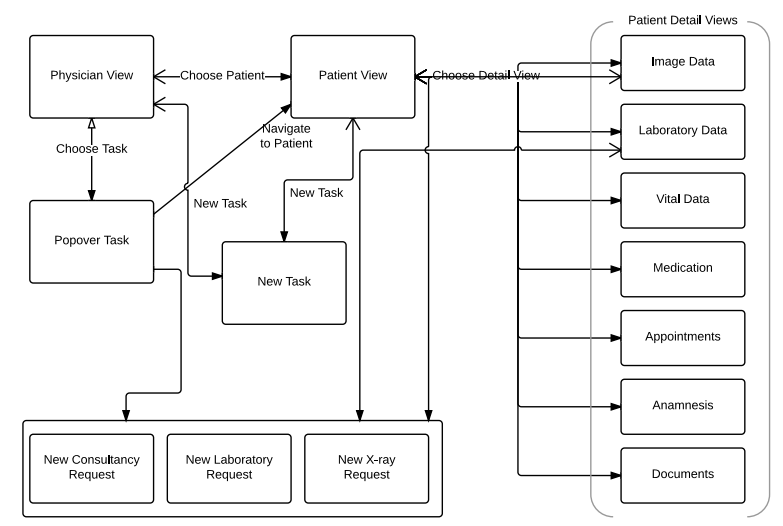

Fig. 10. MEDo Information Architecture

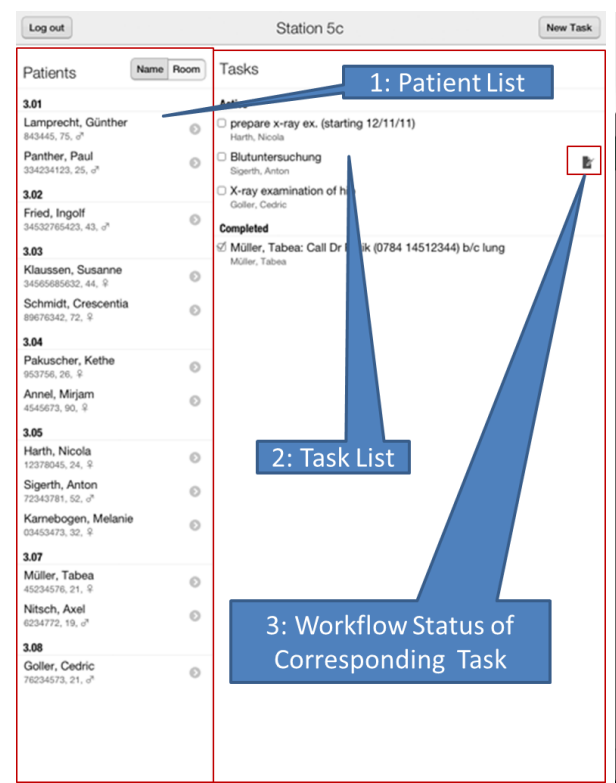

(a) Patient Overview List

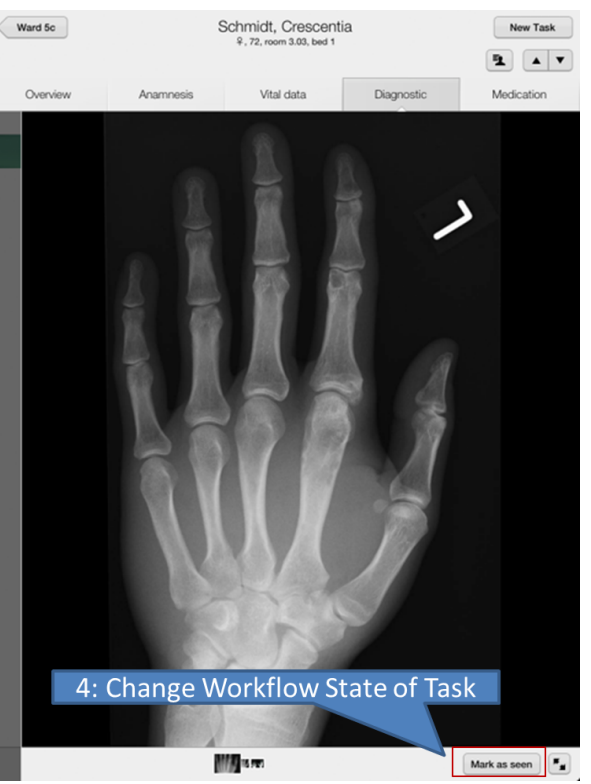

(b) Evaluation of X-ray Image

Fig. 11. Patient Overview List and X-ray Diagnostic View

has turned out to increase task acquisition speed significantly. In addition, related workflows can be automatically derived from task definitions based on these text templates. For example, if a physician uses text template Request Council when defining a task, a corresponding workflow will be started in the background. In addition to text templates, we integrated a voice feature (cf. Marking 6), i.e., the physician may use her voice for recording a task. What is missing at this point, is to derive respective workflows directly from these voice recordings. Fig. 


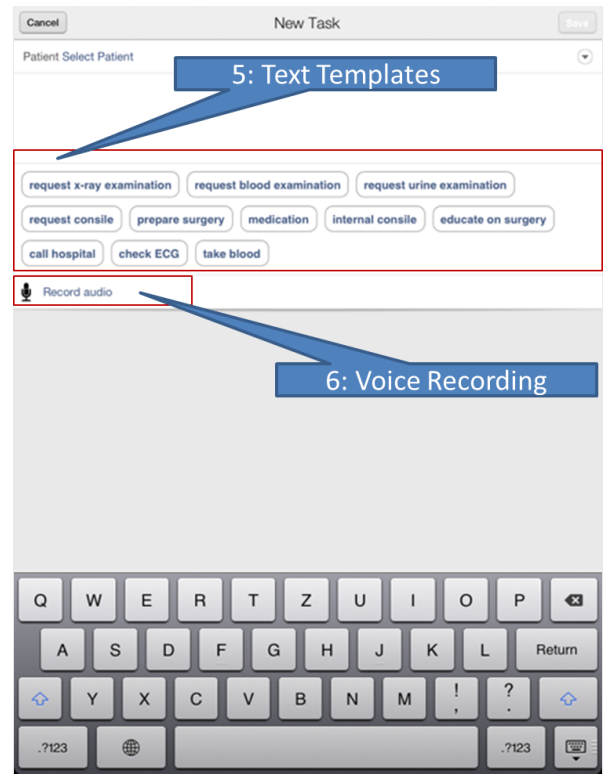

(a) Create Task View

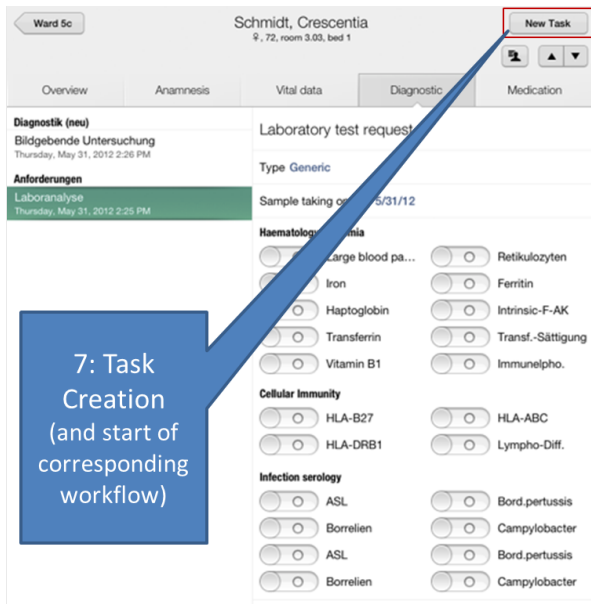

7: Task Creation

(and start of corresponding workflow)

(b) Request for a Laboratory

Fig. 12. MEDo Task Creation and Laboratory Diagnostic View

$12 \mathrm{~b}$ shows the form of a laboratory request. Marking 7 exemplarily shows different ways of creating tasks for the laboratory view. In interviews with physicians and nurses, for every MEDo view we revealed how many creation buttons for tasks are useful and in what way they should be positioned on the screen. The user interface was optimised in several cycles based on the feedback provided by medical members. Finally, after working with MEDo, we interviewed physicians whether they feel comfortable with the MEDo information architecture (see Fig. 13 for results).

\begin{tabular}{lcc}
\hline Question & Mean Value & Standard Deviation \\
\hline View Navigation & 2.00 & 0.47 \\
& 3.67 & 1.66 \\
Patient Communication & 1.90 & 0.50 \\
\multicolumn{2}{c}{ Overall Impression } & | Scale from 1 to 6 | 1: Best Value | 6: Worst Value | \\
\hline \multicolumn{2}{c}{}
\end{tabular}

Fig. 13. MEDo Information Architecture Evaluation

Fig. 14 summarizes all basic requirements for our MEDo information architecture. Requirements $R_{10}-R_{12}$ refer to collaboration among medical staff in connection with their tasks. Obviously, collaboration is crucial in the context of ward rounds and hence must be smoothly integrated with task management. 


\begin{tabular}{lll}
\hline & Information Architecture Requirements & Description \\
\hline $\mathbf{R}_{10}$ & Share the task list with other physicians and nurses & Share tasks with other physicians and nurses. \\
$\mathbf{R}_{11}$ & Provide a multit-user mode for tasks & Provide different views for physicians and nurses. \\
$\mathbf{R}_{\mathbf{R}_{2}}$ & Enable an easy shift management for task lists & Electronic worksheets have to be easily handed over between the staff of a shift. \\
$\mathbf{R}_{\mathbf{1 3}}$ & Provide the user with special views on upcoming tasks & Handling upcoming tasks shall be eased. Access to them must be quick and easy. \\
$\mathbf{R}_{14}$ & Provide the user with views on examination results & A fast and adequate access to examination results is very important. \\
\hline
\end{tabular}

Fig. 14. Information Architecture Requirements

\subsection{Lessons Learned}

When interviewing the physicians, we learned that patient communication suffers from using MEDo; i.e., an intense pursuit with the mobile application distracts physicians from inpatients. Hence, further research is needed to deal with this issue. We further learned that the status of workflows should be presented more intuitively to clinicians. Most of them demand a status notification similiar to traffic lights (i.e., using three colours for presenting status information). Furthermore, physicians missed sub-categories for the pre-defined text templates. Another demand was to integrate more patient information in MEDo (e.g, practitioner information or surgery appointments.). Finally, MEDo must be smoothly integrated into existing hospital information systems.

\subsection{Overall Conclusion}

After using MEDo over a period of four weeks we evaluated it again the same way as described in Section 2. Corresponding results are depicted in Fig. 15.

\begin{tabular}{lcc}
\hline Question & Mean Value & Standard Deviation \\
\hline Task Definition & 1.90 & 0.50 \\
Ward Round Management & 1.80 & 0.53 \\
\hline \multicolumn{2}{r}{ | Scale from 1 to 6 | 1: Best Value | 6: Worst Value | } \\
\hline
\end{tabular}

Fig. 15. MEDo Evaluation

First, physicians conceived the task creation with MEDo on the same level as that of pen and paper (cf. Section 3.1). Second, physicians considered MEDo during wards rounds as useful. More precisely, physicians considered the integration of patient information and workflows as the major issues for using MEDo (cf. Section 3.2). Third, regarding the information architecture, only few issues have been criticised (cf. Section 3.3). For example, the shapes of certain buttons were not intuitively enough in some views. Overall, physicians were satisfied using MEDo when comparing the evaluation results from Figs. 15 and 1.

\section{Related Work}

In $[2,3,4]$, approaches adopting mobile information technology to ward rounds are described. However, none of these approaches is centered around mobile task 
assistance as supported in MEDo. Similar concerns hold for approaches supporting ward rounds by using workflow technology $[5,6,7]$ as well as for approaches transferring information technology to ward rounds [8, 9, 10]. Furthermore, commercial solutions have to be considered, like the one from the orthopaedic ward round (cf. Fig. 2). Finally, much research effort has been spent on the measuring of ward rounds [11, 12]. Overall, none of these approaches centers the solution around a suitable mobile task assistance.

\section{Summary and Outlook}

Existing information technology does not consider requirements of hospital ward rounds adequately. To cope with this drawback, we suggest mobile task management as designed in MEDo as a first approach towards this direction. In further research, we will extend MEDo considering the demands discussed in Section 3.

\section{References}

1. Reichert, M., Weber, B.: Enabling Flexibility in Process-Aware Information Systems: Challenges, Methods, Technologies. Springer, Berlin-Heidelberg (2012)

2. Carlsson, V., Klug, T., Ziegert, T., Zinnen, A.: Wearable Computers in Clinical Ward Rounds, Berlin/Offenbach, Germany. (2006)

3. Lamber, P., Ludwig, B., Ricci, F., Zini, F., Mitterer, M.: Message-based patient guidance in day-hospital. In: Proc. IEEE 12th Int'l Conf. on. MDM '11, IEEE Computer Society (2011) 162-167

4. Kofod-Petersen, A.: Challenges in case-based reasoning for context awareness in ambient intelligent systems. In: 8th European Conf. on CaseBased Reasoning, Ölüdeniz. (2006) 287

5. Ali Fareedi, A., Tarasov, V.: Modelling of the ward round process in a healthcare unit. In: The Practice of Enterprise Modeling. Volume 92 of Lecture Notes in Business Information Processing. Springer Berlin Heidelberg (2011) 223-237

6. Reichert, M.: What BPM technology can do for healthcare process support. In: Proc. AIME'11. Number 6747 in LNAI, Springer (2011) 2-13

7. Wang, L.: An Agent-based Simulation for Workflow in Emergency Department. In: Proc. 2009 IEEE Systems and Information, IEEE (2009) 19-23

8. Bardram, J.E., Bossen, C.: A web of coordinative artifacts: collaborative work at a hospital ward. In: Proc. GROUP '05, ACM (2005) 168-176

9. Ammenwerth, E., Buchauer, A., Bludau, B., Haux, R.: Mobile information and communication tools in the hospital. Int'l Journal of Medical Informatics 57 (2000) $21-40$

10. Pryss, R., Tiedeken, J., Kreher, U., Reichert, M.: Towards flexible process support on mobile devices. In: Proc. CAiSE'10 Forum. Number 72 in LNBIP, Springer (2010) 150-165

11. Alvarez, G., Coiera, E.W.: Interruptive communication patterns in the intensive care unit ward round. I. J. Medical Informatics 74 (2005) 791-796

12. Weber, H., Stöckli, M., Nübling, M., Langewitz, W.A.: Communication during ward rounds in internal medicine. an analysis of patient-nurse-physician interactions using rias. Patient Education and Counseling 67 (2007) 343-348 